\title{
Evaluation of the safety and tolerability of a nutritional Formulation in patients with ANgelman Syndrome (FANS): study protocol for a randomized controlled trial
}

\author{
Donna L. Herber ${ }^{1}$, Edwin J. Weeber ${ }^{2}$, Dominic P. D'Agostino ${ }^{3,4}$ and Jessica Duis ${ }^{5^{*}}$ (1)
}

\begin{abstract}
Background: Ketogenic and low-glycemic-index diets are effective in treating drug-resistant seizures in children with Angelman syndrome. Cognition, mobility, sleep, and gastrointestinal health are intrinsically linked to seizure activity and overall quality of life. Ketogenic and low-glycemic diets restrict carbohydrate consumption and stabilize blood glucose levels. The ketogenic diet induces ketosis, a metabolic state where ketone bodies are preferentially used for fuel. The use of exogenous ketones in promoting ketosis in Angelman syndrome has not been previously studied. The study formulation evaluated herein contains the exogenous ketone beta-hydroxybutyrate to rapidly shift the body towards ketosis, resulting in enhanced metabolic efficiency.

Methods/design: This is a 16-week, randomized, double-blind, placebo-controlled, crossover study to assess the safety and tolerability of a nutritional formula containing exogenous ketones. It also examines the potential for exogenous ketones to improve the patient's nutritional status which can impact the physiologic, symptomatic, and health outcome liabilities of living with Angelman syndrome.
\end{abstract}

Discussion: This manuscript outlines the rationale for a study designed to be the first to provide data on nutritional approaches for patients with Angelman syndrome using exogenous ketones.

Trial registration: ClinicalTrials.gov, ID: NCT03644693. Registered on 23 August 2018. Last updated on 23 August 2018.

Keywords: Ketogenic diet, Ketosis, Angelman syndrome, Placebo-controlled trial, Children, Exogenous ketone, Ketone ester, Ketone supplement

\section{Background}

Angelman syndrome (AS) was first described in 1965 [1], and has a prevalence in the general population of 1 : 10,000-1:24,000 [2-4]. Loss or reduction of function of the maternal $U B E 3 A$ gene (ubiquitin protein ligase E3A) was identified in the late 1990 s as the causative mechanism in the majority of cases [5, 6]. Patients with AS universally have global developmental delays including speech impairment and movement disorders [7]. Individuals are typically described as having a happy disposition with frequent laughter. Eighty percent of patients also

\footnotetext{
* Correspondence: jessica.duis@vumc.org

${ }^{5}$ Division of Medical Genetics \& Genomic Medicine, Department of Pediatrics, Vanderbilt University Medical Center, Nashville, TN 37232-2578, USA Full list of author information is available at the end of the article
}

experience seizures, and all individuals with AS have a disordered electroencephalogram (EEG) $[8,9]$. A large proportion of the population also has feeding problems (75\%) and other gastrointestinal (GI) complaints [7, 10]. Unfortunately, AS has no cure. Typical treatment protocols include pharmacotherapy for seizures, physical and behavioral therapies, and educational interventions [11]. However, approximately $77 \%$ of the AS population experience treatment-resistant seizures [8].

Refractory epilepsy has been treated successfully with specialized dietary approaches, such as the ketogenic (KD), medium-chain-triglyceride (MCT)-based-, and low-glycemic-index diets (LGIT); these diets have also been successful in AS [12-15]. The KD allows the body to shift from carbohydrate-based metabolism to fat- 
based metabolism, thus stimulating hepatic ketogenesis. Nutritional ketosis is a state where the blood levels of ketone bodies are significantly above baseline, typically $>0.5 \mathrm{mmol} / \mathrm{L}$. The ketone bodies (acetoacetate and betahydroxybutyrate (BHB)) are energy substrates used by mitochondria to produce adenosine triphosphate, and can be used as alternative fuels to glucose by the brain, heart, and skeletal muscles.

The high rate of refractory seizures, feeding and GI problems, and severe communication impairments in AS lead to a high unmet medical need. Overwhelming challenges exist to ensuring appropriate diets that serve the complex needs of individuals with AS. Nutritional approaches that promote the body's use of ketones as an alternative fuel may improve overall nutritional status and allow for a liberalized dietary therapeutic strategy in individuals with AS and, therefore, improve quality of life.

Dietary supplementation with ketogenic ingredients is hypothesized to have an important impact on meeting the nutritional ketosis needs of patients with AS. Ketone supplements can aid in achieving and maintaining ketosis, improve the patient's overall nutritional health in AS symptom management, and may improve quality of life for patients and their families. This study is designed as the first-ever to evaluate the safety and tolerability of the ketogenic ingredient beta-hydroxybutyrate in a patient population with a high rate of refractory epilepsy. This manuscript describes the rationale, methods, and potential importance of ketone-based nutritional interventions in children with AS.

\section{Objectives}

The primary objective of the trial is to assess the tolerability of a nutritional formulation containing BHB in patients with AS aged 4-11 years on a variety of dietary backgrounds. The secondary objectives include assessment of ketosis when consuming the nutritional formulation and safety of the nutritional formulation in patients with AS. These outcome measures were chosen as this is a vulnerable, nonverbal population with limited ability to communicate difficulties with tolerability and safety concerns. Additionally, the degree of ketosis attained may inform future trials to assess the beneficial impact of patient nutritional status on health outcomes. All measures are assessed at baseline and the end of each 4-week intervention period (placebo versus test formulation).

\section{Methods/design}

\section{Population and setting}

This trial is conducted by Vanderbilt University Medical Center in collaboration with sponsor Disruptive Nutrition, LLC, and the onsite Angelman Syndrome clinic at the Monroe Carell Jr. Children's Hospital at Vanderbilt is the single study site. The study is funded by the Foundation for Angelman Syndrome Therapeutics (FAST). The research team at Vanderbilt is composed of lead investigator Jessica Duis, MS, MD (pediatric geneticist, Assistant Professor, Division of Medical Genetics and Genomic Medicine, Director, Prader-Willi Syndrome Comprehensive Clinic at Vanderbilt and Director of the Angelman Syndrome Comprehensive Clinic at Vanderbilt), who is responsible for overseeing the trial, including recruitment and taking consent, the majority of clinical assessments of the subjects, and data analysis. Robert Carson, MD, PhD (pediatric neurology and epilepsy specialist, Assistant Professor of Pediatrics at Vanderbilt University), will be the lead neurologist for the study with the responsibility of electroencephalogram (EEG) data interpretation. Fenna Phibbs, MD, MPH (neurology, Associate Professor of Neurology, Vanderbilt Department of Neurology, Vanderbilt University Medical Center) will conduct the mobility tracking. Alexandra Key, MD, PhD (Research Associate Professor of Hearing \& Speech Sciences and Psychiatry \& Behavioral Sciences; Associate Director, IDDRC Translational Neuroimaging Core C; Director, Vanderbilt Kennedy Center Psychophysiology Lab) will be responsible for collection of the event-related potential (ERP) data and analysis. Patience Ergish, MS, RD, LDN (Clinical Dietician at Vanderbilt University), will collect patient diet information during clinic visits. LeeAnna Melton, RN, BSN, CCRP (Research Nurse Specialist III, Division of Medical Genetics at Vanderbilt University) and Lakin Householder (Clinical Trials Associate II, Vanderbilt University) are the study coordinators and point of contact for families enrolled in the trial. This Vanderbilt research team has primary responsibility for data management and analysis, with coordinated efforts from Disruptive Nutrition's scientific staff. The Vanderbilt research team meets on a weekly basis.

Additional oversight and auditing of the trial is provided by Donna Herber, PhD, Chief Science Officer of Disruptive Nutrition, LLC with weekly conferences with members of the Vanderbilt research team. In-person meetings are planned quarterly, with data auditing semi-annually. A medical and research advisory committee has been established and consists of independent experts and key opinion leaders. The committee meets semi-annually. The Data Safety Monitoring Board is discussed in a separate section of this protocol under "Data Monitoring."

Patients are recruited to the Nashville, Tennessee site at Vanderbilt University. Subjects with AS are recruited through the lead investigator's practice, physician referrals, outreach through social media, and parent support groups such as the Foundation for Angelman Syndrome Therapeutics and the Angelman Syndrome Foundation. Subjects are enrolled with the following inclusion criteria: genetically confirmed diagnosis of Angelman syndrome; age 4-11 years; currently on a LGIT, KD 
(conventional 4:1 or 3:1, MCT, modified Atkins), or standard diet (regular diet) consistently for at least 3 months; willingness to consume the investigational formulation; and willingness to undergo protocol examinations at home and clinic visits. Subjects are excluded if they: require parenteral nutrition; have major hepatic or renal dysfunction; have a history of diabetes or have diabetes; are significantly underweight with a body mass index < 18.5; participate in other clinical intervention studies within 1 month prior to entry to the study; are unwilling to comply with the protocol requirements; or have contraindications for the use of ketogenic or lowcarbohydrate diets which, in the opinion of the investigator, may influence a patient's ability to participate in the study. Subjects are permitted to continue using all previously prescribed medications, provided the prescriptions have not changed in the 1 month prior to randomization. Parents/guardians of all eligible subjects must sign informed consent prior to study enrollment, following adequate explanation of the aims, methods, objectives, and potential hazards of the trial by the responsible investigator (see Additional file 3).

\section{Study design}

This investigation is a 16-week, randomized, crossover, double-blinded, placebo-controlled exploratory study of the safety and tolerability of a nutritional formulation containing $d, \quad l$-beta-hydroxybutyrate mineral salts (sodium, calcium, magnesium) (trumacro ${ }^{\mathrm{Tm}}$, Disruptive Nutrition, LLC, Durham, NC, USA) for use with a KD, a LGIT diet, and standard diet in subjects with AS. It is being conducted in accordance with the Declaration of Helsinki and is approved by the Institutional Review Board (IRB) at Vanderbilt University Medical Center (protocol number DUISJ10212017084407). See Fig. 1 (Standard Protocol Items: Recommendations for Interventional Trials (SPIRIT) Figure: schedule of enrollment, interventions, and assessments), Additional file 1 for the SPIRIT 2013 Checklist (recommended items to address in a clinical trial protocol and related documents), and Additional file 2 for the schematic of the trial design.

Members of the Vanderbilt research team, including the lead investigator and clinical trial coordinators, are responsible for obtaining informed consent (see Additional file 3 ). Consent is primarily sought through remote interview prior to scheduling the baseline visit in order to reduce travel burden on patients and families. On the consent form, participants will be asked if they agree to the use of their data and banked blood samples should they choose to withdraw from the trial. Participants will also be asked for permission for the research team to share relevant data with people from the institution taking part in the study as well as regulatory agencies where required. After obtaining informed consent from the parent/caregiver, subjects enter a 2 -week run-in period. At the beginning of the run-in period the natural history and medical history is collected from the subject's parent/caregiver. At baseline, a 3-day recall record of the subject's diet, appetite, GI health, sleep, seizure, mood, and activity level are collected. While one goal of this study is to assess the effects of dietary background on the suitability of the nutritional formulation, there is no requirement for a specific number of patients per diet due to the rarity of the population. Additional study measures, including physiologic, are also collected (Fig. 1).

The study design for this nutritional intervention protocol is a randomized, double-blind, placebo-controlled, crossover study. There are two arms to the study, (1) an investigational formulation period followed by a placebo formulation period, or (2) a placebo formulation period followed by investigational formulation period. Each patient will receive both the investigational formulation and the placebo formulation, with a washout period between each arm. The randomization schedule was created by the Investigational Drug Service Pharmacy using randomization software in a 1:1 ratio with a block size of 4. Participants are enrolled in the next open slot of on the randomization table. The nutritional formulations are coded by the manufacturer lot number and, therefore, the clinical staff is blind to the actual contents of the container (active verses placebo).

Once randomized, subjects start Intervention Period 1, a 4-week period in which they receive nutritional formulation containing $\mathrm{BHB}$ or placebo while maintaining the diet noted at entrance into the study. Home monitoring occurs as indicated in Fig. 1. After 4 weeks of blinded intervention, subjects are evaluated in the clinic and then cease consumption of the ketone formulation or placebo for 4week washout period. Following completion of the washout, subjects enter a second 4-week period in which they receive formulation versus placebo. They will continue the home monitoring throughout the duration of the study. Subsequently, at the completion of the second intervention, they undergo the final scheduled study visit and laboratory sampling. A washout period follows Intervention Period 2, and at approximately week 16 of the study, a follow-up call is made to the family to review symptoms and ensure the patient is not experiencing adverse events during this period. The trial schedule of enrollment, interventions, and assessments is outlined in Fig. 1 (FANS trial schedule of procedures).

\section{Procedures and interventions}

Background information regarding the subject's natural history is collected using a REDCap $\odot$ survey (Copyright 2006-2013 Vanderbilt University. All rights reserved) [16]. Body weight and height are measured at each study 


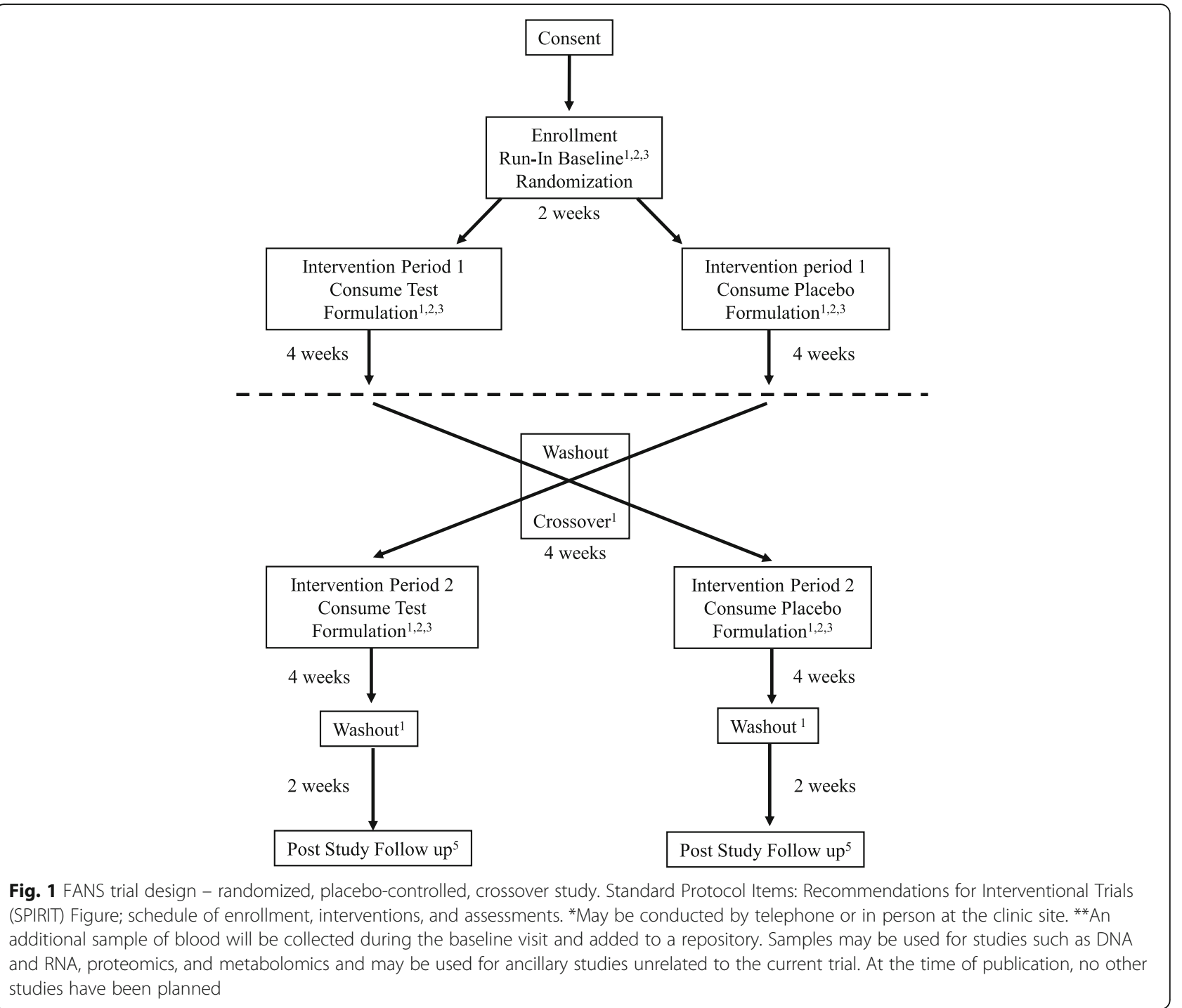

visit. Serum laboratory analysis is conducted to establish a metabolic baseline: comprehensive metabolic panel, complete blood count, ketones (beta-hydroxybutyrate and acetoacetate), and lipid panel. EEG, ERP and gait analysis using the ProtoKinetics Zeno ${ }^{\text {Tn }}$ Walkway are performed at each study visit (Fig. 1). The utility of ERP and gait analysis to predict changes in patient function in an Angelman population has not been clearly established, but their appropriateness in similar populations has been detailed elsewhere $[17,18]$. Questionnaires completed during the study include the Vineland ${ }^{\mathrm{m}}-3$, Developmental Behavioral Checklist (Pearson Education, Inc.), which has been used previously in studies of patients with AS [19], and the Callier-Azusa Scale to assess areas of motor development, perceptual abilities, daily livingskills, language development, and socialization. Dietary intake, seizure frequency, sleep, and GI health are evaluated throughout the protocol through at-home monitoring. Data collected during home monitoring is recorded on tablet devices provided to the parents/caregivers upon enrollment, preloaded with necessary tracking applications. Families are provided an EarlySense $^{\bullet}$ monitor with a written guidance for home sleep monitoring. External sleep monitors are important in an AS population as wearable devices are typically removed by the patient. The monitors have not been studied with patients with AS; however, their validity has been demonstrated in the general population [20]. Urinary ketones are measured by the patient's caregivers throughout the protocol, using Ketostix which are a validated measure of urine acetoacetate [21, 22].

Tolerability is determined at the end of each 4-week intervention period. Protocol compliance is monitored during both investigational and placebo intervention periods by recording the amount of formulation 
consumed. Formulation acceptability is assessed at the end of each intervention period by a parent questionnaire evaluating convenience, taste, and degree of acceptance of the nutritional formulation on a 10-point Likert scale or based on compliance with the study protocol and consumption of the formulation three times daily consistenty during the 4-week interventional period. Similar ranking systems to assess formulation acceptability have been used with other nutritional interventions in epilepsy populations [22-25].

Safety is assessed throughout the entire protocol period through (1) adverse event reporting and (2) assessment of clinical parameters collected throughout the protocol including anthropometrics, blood metabolism profile, dietary intake, seizure frequency, EEG, ERP, mobility, GI function, and sleep habits. In this population, the potential for negative effects of intervention must be closely monitored as patients are nonverbal. Additional file 4 contains details on collecting, assessing, reporting, and managing solicited and spontaneously reported adverse events.

Investigational and placebo formulations are administered with the serving size based on subject body weight. The investigational formulation provides betahydroxybutyrate, $2 \mathrm{~g}$ carbohydrate, $1 \mathrm{~g}$ protein, and $9 \mathrm{~g}$ fat, plus minerals, per $100 \mathrm{kcal}$. The placebo is matched for mineral content only. The formula is given orally as food or beverage three times per day.

Families will be encouraged to continue participation in the trial through weekly telephonic interviews. Data will be collected through three primary interfaces. Background information regarding the patient's natural history will be collected by the clinical staff using the REDCap $\odot$ survey. REDCap $\odot$ is a secure web application that manages the trial database (21 CFR Part 11 and HIPAA compliant). Clinical visits will use either paper or electronic medical records. Data collected during home monitoring will be recorded using a tablet provided to the patient upon enrollment. The tablet is preloaded with the applications necessary to record all data during home monitoring. All data collected, regardless of patient completion status, will be shared with patients upon conclusion of the trial (last patient last visit) and assessment of the data. Additional file 4 contains details on data management and collection procedures.

\section{Statistical considerations}

The primary outcome of the trial is to assess the tolerability of a nutritional formulation containing BHB in patients with AS. Tolerability is demonstrated through patient compliance with the protocol as determined by the amount of formulation consumed as compared to the amount prescribed and is assessed at the end of each intervention period. Premature discontinuation of the nutritional formulation by the family is also deemed as potential intolerability. Tolerability is also assessed at the end of each intervention period through a parental questionnaire. These outcome measures were chosen as this is a vulnerable, nonverbal population with limited ability to communicate and, therefore, determining if the formulation is suitable and well tolerated is an appropriate first step.

The secondary outcomes include assessment of ketosis when consuming the nutritional formulation and safety of the nutritional formulation in patients with AS. Ketosis is evaluated daily through urine testing as well as blood analysis at the end of each intervention period. The degree and timing of ketosis may be clinically relevant to demonstrating better patient nutrition as nutritional ketosis has been related to better patient outcomes as described in the background of this manuscript. Safety is evaluated by changes in motor function, cognitive function, GI tolerance, sleep, and seizures, along with changes in height, weight, and blood metabolic panels as assessed at the end of each intervention period. In addition, adverse event reporting is monitored in real time by the study coordinator.

No studies testing ketone supplementation in patients with AS exist. Therefore, sample-size estimation is based on measures of ketosis. It is assumed from murine preclinical investigations, non-AS clinical studies using $\mathrm{MCT}$ and/or BHB, and low-carbohydrate dietary interventions, that the minimal change of the secondary outcome, degree of ketosis, from the baseline to the end of the fourth week of the investigational formulation intervention period will be $100 \%$ change from baseline (baseline for standard diet is typically $<0.5 \mathrm{mmol} / \mathrm{L}$ of $\mathrm{d}-\mathrm{BHB}$ in blood, or $<5 \mathrm{mg} / \mathrm{dL}$ acetoacetate in urine). The acetoacetate test used is Ketostix (Bayer Corp. Diagnostics Division, Tarrytown, NY, USA) which exhibits a sensitivity of $78 \%$ and a specificity of $96 \%$ when compared to a serum standard of $14.4 \mathrm{ml} / \mathrm{dL}$. Considering a $25 \%$ dropout rate, an estimate of 25 subjects need to be screened to achieve 15 completers.

Descriptive statistics, such as mean and standard deviation, are used to describe continuous variables, and frequency for the categorical variables. Means are compared using a paired two-sample $t$ test. Statistical significance for all tests is $p<0.05$. Multiple comparisons can be made including: (1) each subject to serve as their own control, comparing investigational formulation to placebo formulation, as well as to baseline, (2) each dietary background group considered in aggregate, comparing investigational formulation to placebo formulation, as well as to baseline, and (3) all subjects considered in aggregate, comparing investigational formulation to placebo formulation, as well as to baseline.

Any patient who receives the study formulation for any amount of time will be included in the intent-totreat population for study analyses. Missing data points 
in this population will be addressed in discussion of any published results. Data from protocol-compliant participants is the defined population for efficacy subset analyses; any participant who received both protocolrequired formulations and all required clinical assessments is considered protocol compliant. Such an efficacy subset analysis will be the primary approach for determining whether the trial endpoints are met. Those patients who complete the baseline visit, but do not start Intervention Period 1 and are withdrawn from the study, will be used for natural history reportable data only but will not be included in analyses for determining whether the outcome measures of the trial have been met.

\section{Discontinuation/withdrawal from study participation}

Participation in this study is voluntary. The parent or guardian of a participant may withdraw consent to participate in the study at any time for any reason that they deem necessary. The administration of the study formulation may be discontinued abruptly and does not require any further medical intervention and no alternate therapy will be offered.

\section{Data monitoring}

In order to ensure the safety of the study participants, members of a Data Safety Monitoring Board (DSMB) have been appointed by the principal investigator and Vanderbilt University. The board consist of a chairperson, biostatistician, and three independent reviewers with expertise in the research field. Each member of the board meets these minimum qualifications including: (1) expertise in the field, (2) experience in the conduct of clinical trials and statistical knowledge, (3) independence from the direct management of the clinical trial, and (4) no conflict of interest. The chairperson is responsible for overseeing the meetings, developing the agenda, summarizing the meeting, and is the contact person for the DSMB. The DSMB meets three to four times per year, such schedule to be determined by the chairperson. Additional details on the charter of the DSMB can be had by contacting the Vanderbilt University Medical Center Human Research Protections Program at www.vumc.org/irb.

\section{Ethical considerations}

This study is to be conducted according to US and international standards of Good Clinical Practice (FDA Title 21 part 312 and International Conference on Harmonization guidelines), applicable government regulations and institutional research policies and procedures. This protocol and any amendments will be submitted to the Vanderbilt University IRB in agreement with local legal prescriptions, for formal approval of the study conduct. The DSMB will work in conjunction with the principal investigator and IRB to determine whether a protocol amendment warrants additional patient consent or other communication to the enrolled patients and their families. The decision of the IRB concerning the conduct of the study will be made in writing to the investigator before commencement of this study. Protection of participant confidentiality is described further in Additional file 4.

Participants may be able to claim compensation for injury caused by participation in this clinical trial. Participants who sustain injury and wish to make a claim for compensation should do so in writing in the first instance to the principal investigator, who will pass the claim to the sponsor's insurers, via the sponsor's office.

\section{Discussion}

Angelman syndrome is characterized by refractory epilepsy. In fact, the EEGs of individuals with AS are abnormal when compared to neurotypical controls even in the absence of seizures [26]. Fasting has been recognized as a potential treatment for seizures since ancient times, and dietary intervention that mimicked fasting was introduced in the early 1920s [27, 28]. These diets place the body in a state of nutritional metabolic ketosis. Using nutritional ketosis as a model, many dietary interventions have been studied in both animal models and in human clinical trials for a variety of neurologic disorders.

For children with refractory epilepsy, dietary interventions leading to nutritional ketosis are efficacious and safe. A modified Atkin's diet, prospectively studied in children aged 3-18 years, demonstrated that moderate urinary ketosis developed within 4 days with significant improvement in seizure frequency at 6 months [29]. In AS, case reports also demonstrate efficacy [30]. For a 5year-old girl with uncontrollable daily seizures resistant to triple-anticonvulsant therapy, a KD proved to be effective in diminishing seizures from the first week of initiation. An EEG with no epileptic activity confirmed the patient's improvement along with improvement in sleep and hyperactivity. In a separate study in patients with AS, 4 months of LGIT resulted in decreased seizure frequency with correlative improvement post-trial EEG, with evidence of developmental progress. The diet was well tolerated and five of six subjects remained on the LGIT after completion of the trial [14]. A retrospective medical record review of 23 subjects with AS who utilized LGIT suggested that $22 \%$ of subjects maintained complete seizure freedom, $43 \%$ maintained seizure freedom except in the setting of illness or non-convulsive status epilepticus, and $30 \%$ had a decrease in seizure frequency [13].

The literature suggests a safe transition from glucose to ketone bodies as fuel. A large study of the use of the 
LGIT diet in pediatric epilepsy revealed limited side effects and decreased seizure frequency [31]. Studies of the MCT diet revealed GI complaints as the greatest tolerability issue [32]. Acidosis has also been reported. The risk of nutritional deficiency in children on restrictive dietary treatments and a lack of KD-specific supplements raised concerns about micronutrient status. Vitamins A and $\mathrm{E}$, zinc, selenium, calcium, and magnesium levels have been measured in children with intractable epilepsy treated with the KD [33]. The researchers suggested that changes in plasma vitamins $\mathrm{A}$ and $\mathrm{E}$ and the decline in magnesium status after 12 months may be suboptimal and require supplemental approaches to the KD.

While clinical evidence suggests that nutritional ketosis through diet is effective, well tolerated and safe in seizure control; little clinical evidence is available regarding the consumption of exogenous ketones as part of nutritional support to improve a patient's overall health, supporting dietary and pharmacologic management of seizures and other symptoms. Such an approach is supported by animal models. Transgenic mice, altered at the E6-AP ubiquitin ligase gene which is known to be the causative factor in AS, resemble human AS with motor dysfunction, inducible seizures, and a contextdependent learning deficits $[5,6,34]$. These mice were fed an exogenous ketone (R, S-1,3-butanediol acetoacetate diester) ad libitum for 8 weeks, mixed into the standard rodent chow at $10 \%$ weight/weight. The animals were in nutritional ketosis and demonstrated improved motor coordination, learning and memory, and synaptic plasticity [35]. Exogenous ketone supplements (ketone ester $2.5 \mathrm{~g} / \mathrm{kg} /$ day; BHB salt + MCT: $2.5 \mathrm{~g} / \mathrm{kg} /$ day) administered orally for 7 days to genetic absence-like epilepsy Wistar Albino Glaxo/Rijswijk (WAG/Rij) rats that have modulated absence epileptic activity showed significantly decreased number of spike-wave discharges. These improvements returned to baseline levels on the first day without ketone supplementation [36].

The animal evidence supporting ketone supplementation and the clinical evidence for KDs in models of AS and seizure control, respectively, suggest that dietary interventions including exogenous ketones may be an important development in clinical management strategies. The current study is designed as an initial evaluation of ketogenic formulations used with a variety of dietary backgrounds. It represents a unique opportunity to study the potential benefits of nutritional intervention in a pediatric population (ages 4-11 years) with AS. The knowledge gained from the study may guide further evaluations in the use of exogenous ketones for nutritional strategies in the dietary management of patients AS.

It is known in the AS population that there is a large placebo effect. Our study also offers a unique design in which the use of objective measures is prioritized. We hope that this will yield measures that benefit future trials in the Angelman community in addition to a clearer understanding of the overall effectiveness and mechanism of action of BHB for inducing ketosis. The ultimate goal will be to positively impact nutritional status, which should enhance the ability of the patient to overcome the symptoms of AS such as seizures, cognition and learning, gait/mobility, and/or sleep disturbances.

\section{Trial status}

ClinicalTrials.gov, ID: NCT03644693. Registered on 23 August 2018. Last Updated on 23 August 2018; recruitment start date November 2018; approximate completion date December 2019.

\section{Supplementary information}

Supplementary information accompanies this paper at https://doi.org/10. 1186/s13063-019-3996-x.

Additional file 1. Standard Protocol Items: Recommendations for Interventional Trials (SPIRIT) 2013 Checklist.

Additional file 2. Schematic of trial design.

Additional file 3. Patient consent document.

Additional file 4. Additional protocol details.

\section{Abbreviations}

AS: Angelman syndrome; BHB: Beta-hydroxybutyrate;

EEG: Electroencephalogram; ERP: Event-related potential; Gl: Gastrointestinal; IRB: Institutional Review Board; KD: Ketogenic diet; LGIT: Low-glycemic-index therapy; MCT: Medium-chain triglycerides; UBE3A: Ubiquitin protein ligase E3A

\section{Acknowledgements}

A special thank you to Terry Jo Bichelle, Paula Evans, Allyson Berent, and Meagan Cross for their insight from the family's perspective of living with a child with Angelman syndrome. Their deep knowledge of this condition was invaluable. We are also grateful to Frank Cerasoli, PhD and Sophia Ononye, PhD for their professional writing services.

\section{Trial sponsor}

This trial is sponsored conducted by Vanderbilt University Medical Center in collaboration with sponsor Disruptive Nutrition, LLC, and the onsite Angelman Syndrome Foundation clinic at the Monroe Carell Jr. Children's Hospital at Vanderbilt is the single study site. It is funded by the Foundation for Angelman Syndrome Therapeutics (FAST) in an award granted to the PI, Dr. Jessica Duis.

\section{Authors' contributions}

DLH participated in trial design and drafting the manuscript. EJW and DPD participated in trial design. JD is the principal investigator for this

investigator-driven clinical trial and the point of contact for all trial inquiries; she participated in trial design and drafting the manuscript. All authors read and approved the final manuscript.

\section{Authors' information}

DLH is the Chief Science Officer for Disruptive Enterprises, LLC, of which Disruptive Nutrition, LLC is a wholly owned subsidiary. EJW was formerly Professor, Molecular Pharmacology and Physiology, Chief Scientific Officer, USF Health Byrd Alzheimer's Institute, Director, Murine Neurobehavioral Testing Facility, and Associate Professor, Molecular Pharmacology and Physiology at the University of South Florida. He is currently Vice President, Biology-Neuroscience at PTC Therapeutics. DPD is an Associate Professor, College of Medicine Molecular Pharmacology and Physiology at the University of South Florida and a Research Scientist at the Institution for Human 
and Machine Cognition. JD is an Assistant Professor, Division of Medical Genetics and Genomic Medicine, Director, Prader-Willi Syndrome Comprehensive Clinic at Vanderbilt and Director of the ASF-supported Angelman Syndrome Comprehensive Clinic at Vanderbilt.

\section{Funding}

This project is funded by the Foundation for Angelman Syndrome Therapeutics in collaboration with Disruptive Nutrition, LLC. The Foundation reviewed a grant application substantially equivalent to the protocol described herein, but did not have direct input into protocol design or writing the manuscript. DLH is employed by Disruptive Nutrition and was involved in the design of the study and writing the manuscript. Neither the Foundation nor Disruptive Enterprises has ultimate authority over study design, collection, management, analysis, and interpretation of data; writing of the report, or publication; this responsibility rests with the trial sponsor, Vanderbilt University, and principal investigator, Dr. Duis.

\section{Availability of data and materials}

Data sharing is not applicable to this article as no datasets were generated or analyzed during the current study. The principal investigator and clinical team will have access to the final dataset. The research team at Disruptive Nutrition will only have access to de-identified data.

\section{Ethics approval and consent to participate}

This clinical trial protocol was approved by the IRB at Vanderbilt University in November 2018 (protocol number DUISJ10212017084407). Potential parents/ guardians of participants will be contacted initially by a member of the Angelman syndrome clinic at Vanderbilt University. Parents will be provided with the IRB approved version of the informed consent document (Additional file 3). Participants are children between the ages of 4 to 11 years and, therefore, consent obtained from the person with parental rights after they have read the informed consent and had their questions answered.

\section{Consent for publication}

Consent is not applicable; the publication plan is in Additional file 4.

\section{Competing interests}

DLH is employed by Disruptive Enterprises, LLC which has exclusively licensed the patents to the formula used in this study. EJW is an inventor on one of the patents covering the study formulation, has received research support from the Foundation for Angelman Syndrome Therapeutics, and is currently employed by PTC Therapeutics which engages in the development and commercialization of gene therapies for Angelman syndrome. DPD is an inventor on one of the patents covering the study formulation, and has received funding from Disruptive Enterprises, LLC and the Office of Naval Research. JD has received funding from the Foundation for Angelman Syndrome Therapeutics and the Angelman Syndrome Foundation.

\section{Author details}

'Disruptive Nutrition, LLC, 300 West Morgan Street, Suite 1510, Durham, NC 27701, USA. ${ }^{2}$ PTC Therapeutics, Inc., 100 Corporate Court, South Plainfield, NJ 07080-2449, USA. ${ }^{3}$ Morsani College of Medicine, Department of Molecular Pharmacology and Physiology, University of South Florida, 12901 Bruce B Downs Blvd, Tampa, FL 33612, USA. ${ }^{4}$ Institute for Human and Machine Cognition, Ocala, FL, USA. ${ }^{5}$ Division of Medical Genetics \& Genomic Medicine, Department of Pediatrics, Vanderbilt University Medical Center, Nashville, TN 37232-2578, USA

\section{Received: 11 August 2019 Accepted: 13 December 2019} Published online: 09 January 2020

\section{References}

1. Angelman H. Puppet children. Dev Med Child Neurol. 1965;7:681-8.

2. Williams CA, Beaudet AL, Clayton-Smith J, Knoll JH, Kyllerman M, Laan LA, Magenis RE, Moncla A, Schinzel AA, Summers JA, Wagstaff J. Angelman syndrome 2005: updated consensus for diagnostic criteria. Am J Med Genet A. 2006:1;140(5):413-8.

3. Bailus BJ, Segal DJ. The prospect of molecular therapy for Angelman syndrome and other monogenic neurologic disorders. BMC Neurosci. 2014;15:76.
4. Mertz LG, Christensen R, Vogel I, Hertz JM, Nielsen KB, Grønskov K, Østergaard JR. Angelman syndrome in Denmark. birth incidence, genetic findings, and age at diagnosis. Am J Med Genet A. 2013;161A(9):2197-203.

5. Kishino T, Lalande M, Wagstaff J. UBE3A/E6-AP mutations cause Angelman syndrome. Nat Genet. 1997;15(1):70-3 Erratum in: Nat Genet 1997;15(4):411.

6. Matsuura T, Sutcliffe JS, Fang P, Galjaard RJ, Jiang YH, Benton CS, Rommens $J M$, Beaudet AL. De novo truncating mutations in E6-AP ubiquitin-protein ligase gene (UBE3A) in Angelman syndrome. Nat Genet. 1997;15(1):74-7.

7. Clayton-Smith J, Pembrey ME. Angelman syndrome. J Med Genet. 1992; 29(6):412-5.

8. Thibert RL, Conant KD, Braun EK, Bruno P, Said RR, Nespeca MP, Thiele EA. Epilepsy in Angelman syndrome: a questionnaire-based assessment of the natural history and current treatment options. Epilepsia. 2009; 50(11):2369-76

9. Thibert RL, Larson AM, Hsieh DT, Raby AR, Thiele EA. Neurologic manifestations of Angelman syndrome. Pediatr Neurol. 2013;48(4):271-9.

10. Glassman LW, Grocott OR, Kunz PA, Larson AM, Zella G, Ganguli K, Thibert $\mathrm{RL}$. Prevalence of gastrointestinal symptoms in Angelman syndrome. Am J Med Genet A. 2017;173(10):2703-9.

11. Tan WH, Bird LM. Angelman syndrome: current and emerging therapies in 2016. Am J Med Genet C Semin Med Genet. 2016;172(4):384-401.

12. Pfeifer HH, Lyczkowski DA, Thiele EA. Low glycemic index treatment: implementation and new insights into efficacy. Epilepsia. 2008:49(Suppl 8):42-5.

13. Grocott OR, Herrington $\mathrm{KS}$, Pfeifer $\mathrm{HH}$, Thiele EA, Thibert RL. Low glycemic index treatment for seizure control in Angelman syndrome: a case series from the Center for Dietary Therapy of Epilepsy at the Massachusetts General Hospital. Epilepsy Behav. 2017:68:45-50.

14. Thibert RL, Pfeifer HH, Larson AM, Raby AR, Reynolds AA, Morgan AK, Thiele EA. Low glycemic index treatment for seizures in Angelman syndrome. Epilepsia. 2012;53(9):1498-502.

15. Kumada T, Miyajima T, Oda N, Shimomura H, Saito K, Fujii T. Efficacy and tolerability of modified Atkins diet in Japanese children with medicationresistant epilepsy. Brain and Development. 2012;34(1):32-8.

16. Harris PA, Taylor R, Thieke R, Payne J, Gonzalez N, Conde JG. Research electronic data capture (REDCap)—A metadata-driven methodology and workflow process for providing translational research informatics support. J Biomed Inform. 2009:42(2):377-81.

17. Key AP, Yoder PJ, Stone WL. Consonant differentiation mediates the discrepancy between non-verbal and verbal abilities in children with ASD. J Intellect Disabil Res. 2016;60(5):478-90.

18. Galilee A, Stefanidou C, McCleery JP. Atypical speech versus non-speech detection and discrimination in 4- to 6- yr old children with autism spectrum disorder: an ERP study. PLoS One. 2017:12(7):e0181354.

19. Gentile JK, Tan WH, Horowitz LT, Bacino CA, Skinner SA, Barbieri-Welge R, Bauer-Carlin A, Beaudet AL, Bichell TJ, Lee HS, Sahoo T, Waisbren SE, Bird LM, Peters SU. A neurodevelopmental survey of Angelman syndrome with genotype-phenotype correlations. J Dev Behav Pediatr. 2010;31(7):592-601.

20. Paalasmaa J, Waris M, Toivonen $H$, Leppäkorpi L, Partinen M. Unobtrusive online monitoring of sleep at home. Conf Proc IEEE Eng Med Biol Soc. 2012; 2012:3784-8.

21. Urbain $\mathrm{P}$, Bertz $\mathrm{H}$. Monitoring for compliance with a ketogenic diet: what is the best time of day to test for urinary ketosis? Nutr Metab (Lond). 2016;13:77.

22. Kossoff EH, Dorward JL, Turner Z, Pyzik PL. Prospective study of the modified Atkins diet in combination with a ketogenic liquid supplement during the initial month. J Child Neurol. 2011;26(2):147-51.

23. ClinicalTrials.gov [Internet]. Bethesda (MD): National Library of Medicine (US) 2000 Feb 29 - Identifier NCT03196271. Evaluating the Tolerance, Compliance, Acceptability and Safety of Ketocal 2.5:1 LQ, a Nutritionally Complete Liquid Feed for Use as Part of the Ketogenic Diet (KD) in Children $8+$ Years, Adolescents and Adults With Intractable Epilepsy or Other Disorders Where the KD is Indicated; Retrieved 2019 December 9 from https://clinicaltrials.gov/ct2/show/NCT03196271.

24. ClinicalTrials.gov [Internet]. Bethesda (MD): National Library of Medicine (US) 2000 Feb 29 - Identifier NCT01796574. Feasibility and Tolerability of the Ketogenic Diet in the Treatment of Refractory Status Epilepticus in a Neurointensive Care Unit; Retrieved 2019 December 9 from https:// clinicaltrials.gov/ct2/show/NCT01796574.

25. ClinicalTrials.gov [Internet]. Bethesda (MD): National Library of Medicine (US). 2000 Feb 29 - Identifier NCT01834482. A Randomized, Open-label 
Evaluation of KetoCal ${ }^{\circledR}$ in Initial Combination With the Modified Atkins Diet for the Dietary Management of Intractable Adult Epilepsy. https:// clinicaltrials.gov/ct2/show/NCT01834482. Retrieved May 2018.

26. Leyser M, Penna PS, de Almeida AC, Vasconcelos MM, Nascimento Of. Revisiting epilepsy and the electroencephalogram patterns in Angelman syndrome. Neurol Sci. 2014;35(5):701-5.

27. Peterman MG. The ketogenic diet in epilepsy. JAMA. 1925;84(26):1979-83.

28. Peterman MG. The ketogenic diet in the treatment of epilepsy: a preliminary report. Am J Dis Child. 1924;28:28-33.

29. Kossoff EH, McGrogan JR, Bluml RM, Pillas DJ, Rubenstein JE, Vining EP. A modified Atkins diet is effective for the treatment of intractable pediatric epilepsy. Epilepsia. 2006;47(2):421-4.

30. Evangeliou A, Doulioglou V, Haidopoulou K, Aptouramani M, Spilioti M, Varlamis G. Ketogenic diet in a patient with Angelman syndrome. Pediatr Int. 2010:52(5):831-4.

31. Muzykewicz DA, Lyczkowski DA, Memon N, Conant KD, Pfeifer HH, Thiele EA. Efficacy, safety, and tolerability of the low glycemic index treatment in pediatric epilepsy. Epilepsia. 2009;50(5):1118-26.

32. Liu YM. Medium-chain triglyceride (MCT) ketogenic therapy. Epilepsia. 2008; 49(Suppl 8):33-6.

33. Christodoulides SS, Neal EG, Fitzsimmons G, Chaffe HM, Jeanes YM, Aitkenhead $\mathrm{H}$, Cross JH. The effect of the classical and medium chain triglyceride ketogenic diet on vitamin and mineral levels. J Hum Nutr Diet. 2012;25(1):16-26.

34. Jiang YH, Armstrong D, Albrecht U, Atkins CM, Noebels JL, Eichele G, Sweatt JD, Beaudet AL. Mutation of the Angelman ubiquitin ligase in mice causes increased cytoplasmic p53 and deficits of contextual learning and longterm potentiation. Neuron. 1998;21(4):799-811.

35. Ciarlone SL, Grieco JC, D'Agostino DP, Weeber EJ. Ketone ester supplementation attenuates seizure activity, and improves behavior and hippocampal synaptic plasticity in an Angelman syndrome mouse model. Neurobiol Dis. 2016;96:38-46.

36. Kovács Z, D'Agostino DP, Dobolyi A, Ari C. Adenosine A1 receptor antagonism abolished the anti-seizure effects of exogenous ketone supplementation in Wistar albino Glaxo Rijswijk rats. Front Mol Neurosci. 2017;10:235

\section{Publisher's Note}

Springer Nature remains neutral with regard to jurisdictional claims in published maps and institutional affiliations.

Ready to submit your research? Choose BMC and benefit from:

- fast, convenient online submission

- thorough peer review by experienced researchers in your field

- rapid publication on acceptance

- support for research data, including large and complex data types

- gold Open Access which fosters wider collaboration and increased citations

- maximum visibility for your research: over $100 \mathrm{M}$ website views per year

At $\mathrm{BMC}$, research is always in progress.

Learn more biomedcentral.com/submissions 